\title{
Mediated \\ Communities in the Age of Electronic Communication $^{1}$
}

\author{
KOME - An International Journal of Pure \\ Communication Inquiry \\ Volume 1 Issue 1 p. 46-53. \\ (C) The Author(s) 2012 \\ Reprints and Permission: \\ kome@komejournal.com \\ Published by the Hungarian Communication \\ Studies Assoiciation
}

\section{Gábor Szécsi}

University of Pécs, Faculty of Adult Education and Human Resources, Hungary

\begin{abstract}
The electronically mediated communication has transformed our notion of the relation between place and community. With a greater proportion of our communicative acts taking place via electronic media, physical co-presence, the co-located interpersonal relations are diminishing as determinants of the nature of human interactions. This paper argues that the electronically mediated communication contributes to the construction of new, mediated forms of communities which are based on the interaction or operational synthesis of virtual and physical communities. The appearance of these new forms of communities leads to a new conceptualization of the relation between self and community. The aim of this paper is to show that the medium of the mediatization and new conceptualization of community is a specific pictorial language of electronically mediated communication, the semantic structure of which offers new opportunities to grasp and understand the complex notion of new mediated communities and to adopt the idea of a new global, community building language in local and national communities.
\end{abstract}

Keywords: electronically mediated communication, mediated community, new conceptualization of community, networked individual, pictorial language

\section{Introduction}

Linguistic communication is a creative process which determines our personality and identity through our communication roles forming each other. Moreover, the human mind is indeed a communicative system the structure of which is affected by prevailing technologies of social communication. That is to say, there is a specific inner relation between the communicative

\footnotetext{
${ }^{1}$ This paper is an enhanced and substantially rewritten version of a conference proceeding, published as Conceptual and linguistic convergencies in the space of electronic communication. In: K. Nyíri, ed. Towards a philosophy of telecomunications convergence. Budapest: MTA Press, 2007, pp. 303-307.
} 
structure of our minds and the communication technologies which can be regarded as dominant in certain cultures.

This inner relation can be shown in all major historical changes in dominant communicative technologies, from the change-over to literacy to the development of the dominance of electronically mediated communication. In this essay, I consider the above assumption by the investigation of the effects that are produced on our conceptualization of community by the use of electronic media. To clarify the theoretical foundations of this new conceptualization, I take the hypothesis as a starting point that the expansion of electronic communication technologies - including both monologic technologies (radio, television, the internet) and dialogic technologies (mobile telephone) - has transformed our notion of the relation between place and community. With a greater proportion of our communicative acts taking place via electronic media, physical co-presence, the co-located interpersonal relations are diminishing as determinants of the nature of human interactions.

It seems that in the space of electronic media, community should be understood as a mediated network of interactions between individuals who uniformly accept and apply some rules for the communicative actions aiming at the effective exchange of information. In other words, there is an inner relation between the criteria of community and the global and local conditions for an effective method of information exchange. And these global and local conditions transform our notions surrounding the structure and life of community.

The electronically mediated communication, as an inherent part of real life in today's world, contributes to the construction of new, mediated forms of communities which are based on the interaction or operational synthesis of virtual and physical communities. The appearance of these new forms of communities leads to the new conceptualization of the relation between self and community. In the age of electronically mediated communication, the essence of community is a kind of networked individualism in which the networked individuals can chose their own communities, rather than are fitted into them with others involuntarily. Therefore the new, mediated form of community implies an individual-center existence and weaker social ties. The new technologies foster communication links outside the individuals' immediate social surrounds.

Accordingly, electronic communication creates a new context in which our notions of culture, community, society, human interactions become more complex. These more complex notions can be regarded as the bases of the idea of the global and local information communities in which the communication attitudes of a person are determined by their impression of their self as permanently available individual whose communicative acts are embedded in a special information net.

The aim of this paper is to show the basis and effects of this process by examining the following propositions:

1. The expansion of electronically mediated communication leads to the appearance of new, mediated forms of communities.

2. The medium of the mediatization and new conceptualization of community is a specific, pictorial language of electronically mediated communication. 


\section{Towards a new conceptualization of communities}

Our conceptualization of community, when transformed by the use of electronic media, is embedded strongly in the associative system of conceptual relations that represent the network of various communicative acts, that is the various situations of information exchange. With such a conceptualization of mediated community is conceived as a network of communicative interactions. I want to argue here that the ways of understanding of the interactions between virtual and physical communities move beyond the traditional sociological conceptualization of community-as-interpersonal towards a conceptualization of mediated communities that are based on the interaction or the operational synthesis of virtual and physical communities.

Just as traditional theories of community regard community and society as distinct forms, it is also easy to consider physical and virtual communities as mutually exclusive forms of social organization. In this view, physical community can exist only by virtue of physical colocation in space, and is based on people's natural association through sameness and residential solidarity. Virtual communities created by electronically mediated communication, however, attempt to break some of the boundaries of geographic location, gender, and ethnicity established in physical communities. In other words, physical communities are based on shared social and physical boundaries, whereas virtual communities are based on shared social practices and interest.

Considering the influence of mediated communication on our community-concept, however, many theorists believe that we need a synthesis of physical and virtual communities in order to truly inhabit our experiences. Castells (2000), for example, holds that we need a "bridge" between physical and virtual places in order to unify our experience, because virtual communities only deal in fragmented individuals when they are opposed to real life. Others, like Etzioni (2001), Walls (1993), Katz and Rice (2002), Katz et al. (2004), Katz (2007), Haythornthwaite (2007), and Haythornthwaite and Kendall (2010) emphasize that the best communities are indeed the hybrids of physical and virtual communities. They see the ideal communities as virtual communities enhancing physical communities. According to Haythornthwaite and Kendall (2010), online interactions not only have positive effects for physical, place-based communities, but the intersection between online communication and offline world also forms two halves of a support mechanism for communities. For Katz et al. (2004, p. 362), since the electronically mediated communication becomes inherently part of real life in today's world, "we need an operational synthesis of virtual and physical communities in order to have fulfilling, embodied experiences all of the time". In this view, in the age of electronically mediated communication, the dividing line between virtual and physical communities becomes increasingly indistinct. Therefore, as Poster (2001) shows it, the mediated individuals imagine their virtual communities as real. That is, the role of communication as meaningful and value-based in virtual communities also works to construct physical communities as well.

It is obvious that with this new synthesis of virtual and physical communities, electronically mediated communication contributes to a new construction of the self. The mediatization of communities leads to fractured and fragmented selves, because it opens up many other possible communities in which to participate. The new communication technologies enable individuals to participate in alterior systems of value, belief, and desires. As Gergen (2003, p. 111) notes: "New affective bonds are created outside one's social surrounds. The result is that the centered sense of a bounded self slowly gives way to a "multiphrenia" of partial and conflicted senses of self. Identity becomes fluid, shifting in a chameleon-like way from one social context to another". 
Thanks to these changes, the networked individual is attached to the place and position appointed by his own social ties less and less. Through his multi-channel communicative acts he can become acquainted with more and more communal forms, ways of life, traditions and values in the light of which he can choose more deliberately from among the competing local communities. And this more deliberate choice becomes a part of the more and more complex and multi-layered identity of the networked individual. As Meyrowitz (2005, p. 28) writes on the multiple, multi-layered, fluid, and endlessly adjustable senses of the media-networked individuals' identity:

\begin{abstract}
"Rather than needing to choose between local, place-defined identities and more distant ones, we can have them all, not just in rapid sequence but in overlapping experiences. We can attend a local zoning board meeting, embodying the role of local concerned citizen, as we cruise the internet on a wireless-enabled laptop enacting other, non-local identities. And we can merge the two as we draw on distant information to inform the local board of how other communities handle similar issues and regulations. All the while, we can remain accessible to friends, family, and colleagues from anywhere via a text-message enabled mobile phone."
\end{abstract}

By using the electronic communication technologies, a networked individual becomes a part of a network of interactions between humans who uniformly accept and apply some rules for the communicative acts aiming at the effective exchange of information. In other words, the media-networked individuals become members of a virtual community that is determined both by the global and the local conditions for an effective method of information exchange. Regarding the conceptualization of this new virtual community, Green (2003), for example, argues for a new view of community, in which the significance of locality and interpersonality recedes to the benefit of symbolic processes. As Green (2003, p. 55) points out: "As is the case with internet and 'virtual' communities then, understandings of mobile 'communities' should move beyond the conceptualisation of 'communities-as-interestgroups' (secured via the authentication of the embodied liberal individual and their 'right to privacy'), and indeed beyond a traditional sociological conceptualisation of 'communities-asinterpersonal-and-co-located' (secured via relations based on face-to-face interaction in kinship or social commonality). Rather, we should move towards a conceptualisation of 'communities-as-trust-processes' (secured via the mutual, reciprocal and multiple negotiation of mediated, interpersonal, and organization uncertainty and risk.)"

This new conceptualization moves beyond the traditional definition of community, according to which, as Green (2003, p. 53) writes, community "as an ideal type of relation corresponding to "natural will", is distinguished by an appeal to a totality of cultural history in the collective memory of tradition, is defined through common property, family, custom and fellowship, and is bound by consensus, language and ritual".

The basis of this conceptualization is a complex system of associative conceptual relations that includes our concept of community, and integrates the conceptual representations of human interactions which determine the life of community both in a direct and indirect way. The medium of the new conceptualization is a specific pictorial language, the semantic structure of which offers new opportunities to grasp and understand the complex concept of community. 


\section{The pictorial language of electronically mediated communication}

One of the most important criteria of the new, more deliberate attachment to the local is the deliberate application of the ways of usage that create new local communities in the age of electronic communication. And these new ways of usage are rooted in the communication language of electronic media that can be regarded as a result of the convergence of the characteristics of oral and written communication. Thus we consider the new linguistic culture of electronic communication as one of the most important conditions of the conceptual and the social convergences experienced in the space of electronic media. It seemed that this new linguistic culture is the basic both of the global perspective created by electronic communication and the cohesion of the new, mediated communities that are strengthened by deliberate choices of the networked individuals.

In this new linguistic culture, the original social function of language, namely, the building and maintaining of the cohesion of human community, becomes important development, because in the print societies, language has got far from this original function as a consequence of the appearance of the oral-literal bilingualism and the linguistic asymmetry that is rooted in the social dominance of the standard dialect of literacy. That is, instead of strengthening the community cohesion, this bilingualism and asymmetry disintegrates primary human communities since the use of their own dialects is overtly stigmatized in the light of the socially preferred standard dialect of literacy. This communication culture that forces the whole society and all communities to use one preferred language variety goes against individual biologically encoded need of belonging to a primary community.

The original social function of language, however, has survived this linguistic asymmetry developed in print societies. People hold to their everyday use of language, even if they judge their own dialectical varieties incorrect under the pressure of the overt prestige of the standard. Since the members of small local communities generally communicate with one another orally, the linguistic conventions characterizing these communities have survived in the age of standard linguistic varieties too. In these small local communities the importance of cohesion-strengthening, local values outstrips the external social values that are symbolized by the standard forms of written communication. This phenomenon is experienced especially in small, isolated rural and suburban communities where the prestige of the non-standard variety of usage can be regarded, at the same time, as a symbol of communal identity.

In the space of electronic communication, literacy that generated the asymmetry of linguistic norms of oral and written communication seems to be losing of its power and the prestige of the identity-strengthening ways of usage characterizing small communities and group grows. The expansion of the non-standard varieties of language preferred by the networked individuals is accelerated by the use of electronic media (internet, mobile telephone). This process leading to decline of the prestige of literacy is accelerated by the convergence of the oral and the written communication technologies that affects usage since the new kind of orality created by the use of radio and television or, especially, use of multimedia messaging, the synchronous-complementary transmission of speech, text and pictures in the space of mobile and internet communication.

There are well-perceptible, concrete signs of this convergence of the features of the oral and the written usage. Let us consider the texts that are mediated by the internet or mobile telephone! The texts of many e-mail and SMS message actually belong to the domain of speech and not to the domain of written texts. The grammatical and stylistic characteristics of these messages can be regarded as the marks of a special kind of oral communication. These grammatical and stylistic elements, however, are integrated into the texts mediated by new communication technologies more deliberately than into the oral utterances. By using these 
elements, the utterer intends to show that he wishes to accept and apply the norms and the rules of a linguistic community organized by e-mail and SMS communication. That is to say, he uses these grammatical and stylistic elements of linguistic communication to make it unambiguous that he is attached to a community accepting some forms of usage and that this attachment is a consequence of a deliberate choice.

The linguistic forms accepted in this way affect the everyday usage strongly. The use of the special linguistic forms of the texts mediated by the new communication technologies lives its mark on the written communication and leads to the convergence between orality and literacy. What can be regarded as an outcome of this process is the increasingly indistinct dividing line between then linguistic characteristics of oral and written communication. A new communication language is in the making which integrates the forms of language used in oral utterances and in written texts.

The appearance of the new language of communication can be regarded as a consequence of the networked individuals' deliberate choice who want to join in the global information exchange successfully and to express conceptual relations and emotions as a member of a small community by using the new linguistic forms. One of the most characteristic features of this culture is that the advent of multimedia communication has resulted in a strong interaction between picture and language in the process of oral and written messaging.

As multimedia technology expands, the dividing line between the linguistic characteristics of oral and written communication becomes increasingly indistinct. This means that though the syntactic features and structure of this new language of communication remind us of the linguistic world of oral communication, the new language seems to be more complex in terms of its semantic characteristics. Accordingly, the multimedia integration of verbal and pictorial elements, or the convergence of the linguistic features of oral and written communication, contributes to the transformation of the structure of the mind and the content of thought by establishing a new communication culture.

Consequently, by using the term "pictorial language", I am referring not only to the integration of verbal and pictorial components of information exchange, but to the linguistic medium of the specific synthesis of the features of conceptual and pictorial thought. The pictorial character of the language of electronically mediated communication is rooted in the fact that this language includes expressions which refer to complex conceptual relations without a conceptual analysis of them. In other words, a new metaphorical language is in the making, the function of which is to "show" the world rather than to analyze it.

By using this language, we want to "make perceptible" the complexity of conceptual relations to which we refer. The main intention of a user of this kind of language is to embed some conceptual relations in the system of more complex conceptual representations by using words that are suitable for making the complexity of newly-revealed conceptual relations intelligible. This kind of usage leads, on the one hand, to the appearance of new terms in language and, on the other hand, to the novel use of available linguistic elements. In the latter process, the meanings of some words multiply with more and more conceptual relations.

This is why the usage of the word "community" entails the intention of understanding of the overall or global criteria of community in the age of electronic media. These criteria can be attributed to the specific features of communicational space which is globalized by the use of television, the Internet, and mobile telephones. In other words, there is an inner relation between these criteria and the global conditions for an effective method of information exchange. These global conditions (a common information basis, collective trust relations, etc.) transform our notions surrounding the structure and life of community.

Focusing on the inner relation between the new conceptualization of the criteria of community and our notions regarding the global conditions for an effective method of 
information exchange, we can suggest, for example, the following definition: community is a network of interactions between individuals who uniformly accept and apply some rules for the communicative acts aiming at the effective exchange of information.

Of course, our complex notion of community urges us to form many other definitions. And it is obvious that these definitions approach the community-organizing role of information in different way. They have, however, one thing in common: they all must be founded on the analysis of the conceptual and linguistic changes that transform the structure of our minds in the mediated communities of the electronic era. Because these linguistic changes can be regarded as bases of the mediatization of communities and the adopting of the idea of a global, community building language in the new media space. But what kind of language would best serve as a global language in the network of mediated communities? Etzioni (2008), for example, argues for adopting English as a shared, secondary global language in the information age. As Etzioni (2008, p. 124) points out: "a key element of building a global community atop local communities requires that the various nations involved choose the same second language". This second language, of course, does not replace the particularistic, identity constituting primary languages of local and national communities, rather it is best considered as an additional language. Nevertheless an opposition can be experienced to adopting such an additional language in many nations. According to Etzioni (2008, p. 124), "this opposition often conflates preventing English penetration into the primary language with resisting it as second language". Whereas this opposition, as Etzioni (2008, p. 124) writes, " delays overcoming the "babel" effects at great cost to the transparency of global laws, the promotion of shared understandings, and the efficiency of economic transactions".

In my view, as a global process, the appearance of the pictorial characteristic of primary communal languages can contribute to the adoption of an additional global language, because this process, as we have seen, creates the foundations of the convergences of different usages and languages. The global expansion of the pictorial language of electronic communication can be regarded as the basis of the idea of a global information community in which the communication attitudes of a person are determined by his impression of his self as a permanently available person whose communicative acts are embedded in a global information net. In other words, it is by accelerating and mediating linguistic changes leading to a complex notion of global community that electronically mediated communication becomes a source of, as Meyrowitz (2005, p. 30) writes, the "fusion of local and global identities" and, thus, the adoption of the idea of a secondary global language in new, mediated communities.

\section{Conclusions}

This essay holds that in the information age, the medium of the mediatization and new conceptualization of community is a specific, pictorial language of electronically mediated communication. The global linguistic changes traceable to the use of electronic communications technologies lead to a linguistic galaxy which can contribute to the development of higher level of human cohesion. This paper adopts the assumption that through the appearance of this linguistic galaxy, a new, global community comes into existence which is based on the operational synthesis of virtual and physical communities. As demonstrated, with the worldwide expansion of the new communication culture, the original social function of language, namely, the building and maintaining of the cohesion of human communities, becomes important development. 


\section{Bibliography}

Castells, M., 2000. The information age Vol. I: Rise of the network society. Malden, MA: Blackwell.

Etzioni, A., 2008. A global, community building language? International Studies Perspectives, 2, pp.113-127.

Etzioni, A., 2001. The monochrome society. Princeton, NJ: Princeton University Press.

Gergen, K.J., 2003. Self and community in the new floating worlds. In K. Nyíri, ed. Mobile democracy: Essays on society, self and politics. Vienna: Passagen Verlag, pp.103-114.

Green, N., 2003. Community redefined: Privacy and accountability. In K. Nyíri, ed. Mobile communication: Essays on cognition and community. Vienna: Passagen Verlag. pp. 43-56.

Haythornthwaite, C., 2007. Digital divide and e-learning. In: R. Andrews and C. Haythornthwaite, eds. The handbook of e-learning research. London: Sage, pp.97-118.

Haythornthwaite, C. and Kendall, L., 2010. Internet and community. American behavioral scientist, 53 (8), pp. 1083-1094.

Katz, J. E. and Rice, R.E., 2002. Social consequences of internet use: Access, involvement and interaction. Cambridge: MIT Press.

Katz, J. E., Rice, R.E., Acord, S., Dasgupta, K. and David, K., 2004. Personal mediated communication and the concept of community in theory and practice. In R. Kalbfleisch, ed. Communication and community: Communication yearbook 28. Mahwah, NJ: Erlbaum, pp.315-371.

Katz, J.E., 2007. Mobile media and communication. Communication monographs, 74 (3), pp.389-394.

Meyrowitz J., 2005. The rise of glocality: New senses of place and identity in the global village.

In: K. Nyíri, ed. Sense of place: The global and the local in mobile communication. Vienna: Passagen Verlag, pp.21-30.

Poster, M., 2001. What's the matter with the internet? Minneapolis MN: University of Minnesota Press.

Walls, J., 1993. Global networking for local development. In: L.M. Harasim ed. Global networks: Computers and international communication. Cambridge: MIT Press, pp.153-166. 